\title{
Edema pulmonar unilateral. La importancia de la exploración física y la ecografía a pie de cama
}

\author{
Leonor Hernández1', Sonia Marín², Irene Zamora', Alicia Pérez-Bernabéu', José María Cepeda-Rodrigo ${ }^{1}$ \\ ${ }^{1}$ Servicio de Medicina Interna. Hospital Vega Baja. Orihuela (Alicante). España \\ ${ }^{2}$ Servicio de Medicina Interna. Hospital General Universitario de Elda. Elda (Alicante). España
}

Recibido: 18/03/2019

Aceptado: 20/03/2019

En línea: 30/04/2019

Citar como: Hernández L, Marín S, Zamora I, Pérez-Bernabéu A, Cepeda-Rodrigo JM. Edema pulmonar unilateral. La importancia de la exploración física y la ecografía a pie de cama. Rev Esp Casos Clin Med Intern (RECCMI). 2019 (Abr); 4(1): 36-38. doi: 10.32818/reccmi.a4n1a13.

Autor para correspondencia: José María Cepeda-Rodrigo. jmcepedarodrigo@gmail.com

\section{Palabras clave \\ $\triangleright$ Insuficiencia cardíaca \\ $\triangleright$ Ecografía \\ $\triangleright$ Miocardiopatía dilatada}

\begin{abstract}
Resumen
Según datos del informe RECALMIN 2017, el diagnóstico principal al alta más frecuente en las unidades de Medicina Interna es el de insuficiencia cardíaca. Su historia natural se caracteriza por episodios de descompensación, que aumentan la morbilidad y la mortalidad, siendo los signos y síntomas de congestión las principales razones por las que acuden a Urgencias. Presentamos el caso de un paciente sin cardiopatía estructural conocida que ingresó por un episodio de disnea. El objetivo es mostrar la importancia de la exploración física y la ayuda de la ecografía a pie de cama para alcanzar un buen enfoque clínico del paciente e iniciar el tratamiento adecuado.

Abstract
According to data from the RECALMIN 2017 report, heart failure is the most frequent discharge diagnosis in
Internal Medicine Units. Its natural history is characterized by episodes of decompensation, which increase mor-
bidity and mortality, with the signs and symptoms of congestion being the main reasons why they go to the
emergency room. We present a case of a patient without known structural heart disease. The objective is to
demonstrate the importance of the initial physical examination and point of care ultrasound that allows a good
approach to the patient and initiation of adequate treatment.
\end{abstract}

\section{Puntos destacados}

$\triangleright$ El ritmo de galope ventricular tiene una especificidad muy alta para el diagnóstico de insuficiencia cardíaca con función sistólica deprimida.

$\triangleright$ La ecografía clínica aporta información inmediata sobre la existencia de cardiopatía estructural y el estado de congestión.

\section{Introducción}

Según datos del informe RECALMIN publicado en 2017, el diagnóstico principal al alta más frecuente en las unidades de Medicina Interna es el de insuficiencia cardíaca (IC), con un peso de casi un 10\% sobre el total y con un incremento del $32 \%$ en la última década. Del total de altas dadas en los servicios de Medicina Interna en 2015, 66.572 fueron por este motivo'. La historia natural de esta enfermedad se caracteriza por episodios de descompensación aguda, que se asocian con un aumento de la morbilidad y la mortalidad, siendo los signos y síntomas de congestión las principales razones por las que los pacientes con IC aguda buscan atención médica urgente².

Presentamos el caso de un paciente sin antecedentes de hipertensión arterial, diabetes, dislipidemia, ni cardiopatía estructural conocida, que ingresó por disnea e infiltrado pulmonar unilateral. El objetivo es mostrar la importancia de la exploración física complementada con la ecografía a pie de cama para conseguir, desde el momento de la valoración inicial, un buen enfoque clínico del paciente y comenzar un tratamiento adecuado.

\section{Historia clínica}

Paciente varón de 57 años que consultó en Urgencias por disnea de moderados esfuerzos (NYHA II) de un año de evolución, con empeoramiento los días previos hasta convertirse en disnea de mínimos esfuerzos (NYHA III), 
Hernández L, Marín S, Zamora I, Pérez-Bernabéu A, Cepeda-Rodrigo JM. Edema pulmonar unilateral. La importancia de la exploración física y la ecografía a pie de cama

bendopnea y ortopnea. Se acompañaba de tos escasa con expectoración blanquecina, sin dolor torácico ni fiebre. Era fumador activo de 15-20 cigarrillos al día, con un índice paquetes/año de 34, y consumidor enólico de 44 unidades de bebida estándar semanales. No llevaba ningún tratamiento crónico.

\section{Pruebas complementarias}

En Urgencias, se realizaron las siguientes pruebas:

- Radiografía de tórax. Véase Figura 1.

- Electrocardiograma. Mostraba un ritmo sinusal a 110 latidos por minuto, eje a 60 grados, conducción auriculoventricular normal, hipertrofia ventricular izquierda, sin signos de isquemia ni necrosis.

- Bioquímica. Glucosa $148 \mathrm{mg} / \mathrm{dl}$, urea $32 \mathrm{mg} / \mathrm{dl}$, creatinina 0,98 mg/dl, sodio $135 \mathrm{mmol} / \mathrm{l}$, potasio $3,8 \mathrm{mmol} / \mathrm{l}$, transaminasas normales, colesterol total 194 mg/dl, LDL-colesterol 131 mg/dl, albúmina 3,8 g/dl, TSH 1,48 mlU/l, troponina 0,05 ng/ml, NT-proBNP 8.020 pg/dl, Ca-125 124 U/ml, PCR 2,5 mg/dl. El hemograma y la gasometría venosa resultaron normales. No presentaba proteinuria en el uroanálisis. La PCR de gripe A y B fue negativa.

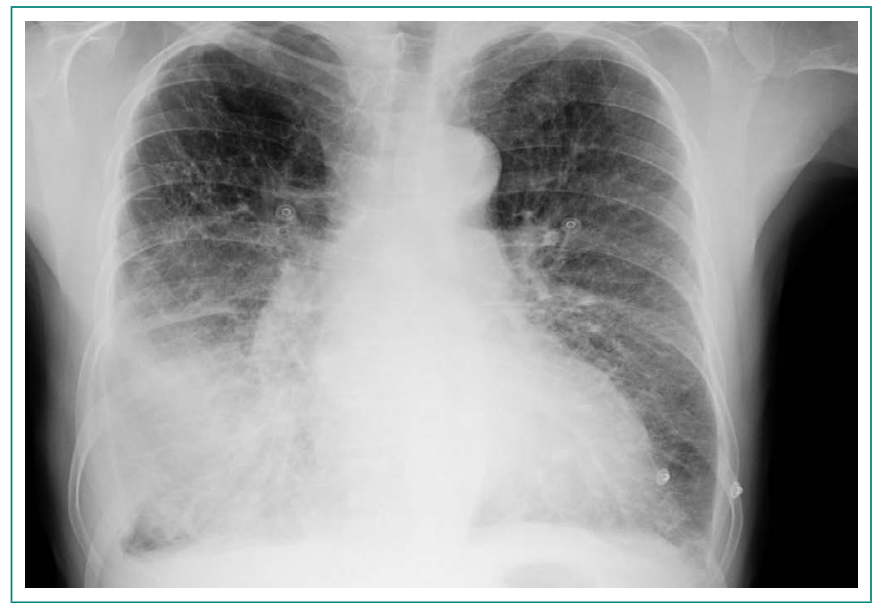

Figura 1. Radiografía de tórax realizada en Urgencias

El paciente ingresó en planta de Medicina Interna con diagnóstico de neumonía adquirida en la comunidad y tratamiento antibiótico empírico con levofloxacino y broncodilatadores. Exploración física al ingreso en planta: temperatura $36,3^{\circ} \mathrm{C}$, presión arterial 220/130 mmHg, saturación basal de oxígeno $95 \%$, frecuencia cardíaca 125 latidos por minuto. No ingurgitación yugular. Tonos rítmicos con ritmo de galope ventricular. Crepitantes bilaterales, de predomino en base pulmonar derecha. La exploración abdominal era anodina y no presentaba edemas ni signos de trombosis venosa profunda en miembros inferiores.

Ante el hallazgo en la auscultación de ritmo de galope ventricular, se realizó en el mismo acto, a pie de cama, una ecografía pulmonar, con hallazgo de un síndrome intersticial pulmonar por presencia de líneas B bilaterales; y una ecocardioscopia, que mostró una disfunción sistólica ventricular izquierda severa (fracción de eyección [FE] estimada del 30\%) con hipocinesia global. Por tanto, cumplía criterios de IC con FE deprimida según la guía de IC de la European Society of Cardiology (ESC) ${ }^{2}$ : signos y síntomas y $\mathrm{FE}<40 \%$. Tanto la auscultación cardíaca, con el hallazgo de ritmo de galope ventricular, como los resultados ecográficos permitieron de manera inmediata un nuevo enfoque diagnóstico del paciente y, como consecuencia, un tratamiento adecuado.

\section{Evolución}

Se suspendió el tratamiento antibiótico y broncodilatador y se inició tratamiento deplectivo con rápida respuesta clínica y radiológica, como se pudo comprobar en la radiografía de tórax repetida a las 24 horas (Figura 2). El ecocardiograma reglado confirmó una miocardiopatía dilatada con disfunción sistólica severa (FE 29\%) por hipocinesia global, hipertrofia ligera de predominio septal y ligera trabeculación de segmentos medioapicales de cara inferior. Presión capilar pulmonar por E/e'19. Ventrículo derecho no dilatado con función normal. Insuficiencia mitral e insuficiencia aórtica leves e hipertensión pulmonar ligera.

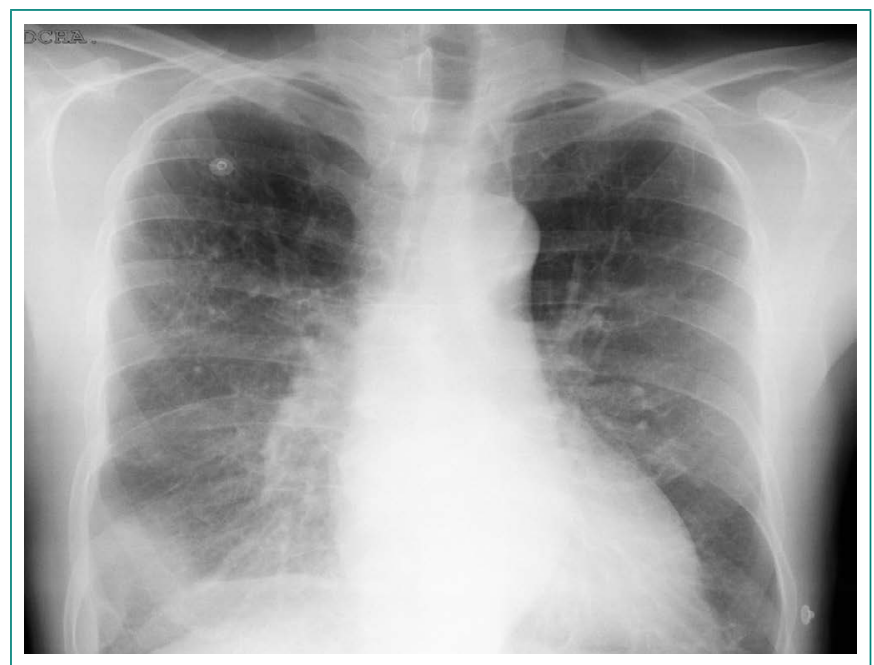

Figura 2. Radiografía de tórax tras 24 horas de tratamiento deplectivo

El paciente fue dado de alta con diagnóstico de IC con FE deprimida por miocardiopatía dilatada de probable origen enólico e hipertensión arterial. Se indicaron cambios en el estilo de vida consistentes en abandono total de hábitos tóxicos (enolismo y tabaquismo) y se inició tratamiento con betabloqueantes, inhibidores de la enzima conversora de angiotensina (IECA), dosis bajas de espironolactona y furosemida.

Fue revisado en consulta de IC 2 semanas más tarde, confirmándose la ausencia de signos congestivos, tanto clínicos como en la ecografía pulmonar, en la que no se encontraron líneas B ni derrame pleural.

\section{Discusión y conclusiones}

La disnea es una de las principales causas de consulta en Urgencias. Hacer un diagnóstico diferencial correcto y seleccionar un tratamiento temprano adecuado supone un desafío clínico, dadas las múltiples patologías que pueden cursar con este síntoma. En nuestro caso, el paciente consultó por disnea con presencia radiológica de edema pulmonar unilateral (EPU), interpretado en el servicio de Urgencias como una neumonía.

El hallazgo radiológico habitual del edema agudo de pulmón cardiogénico es una opacidad bilateral simétrica perihiliar que da lugar a la clásica imagen en alas de mariposa. El EPU es una entidad rara, supone el 2\% del edema pulmonar cardiogénico ${ }^{3}$. Puede ser confundido con otras causas de infiltrados unilaterales en la radiografía de tórax, especialmente con la neumonía, lo que retrasa el tratamiento adecuado. Los pacientes con EPU tienen un riesgo de mortalidad mayor que el edema pulmonar bilateral. En un estudio retrospectivo, la mortalidad del EPU fue del $39 \%$ y la del edema pulmonar bilateral del $8 \%$ 
(odds ratio, 6,9; intervalo de confianza 95\%, 2,6 to 18; $\mathrm{P}<0,001$ ). En los pacientes con EPU, el uso de antibióticos y el retraso en instaurar el tratamiento correcto fueron mayores, lo que puede ser una explicación para esta mayor mortalidad³.

La insuficiencia mitral severa es la causa principal de EPU y la mayor parte de los casos (90\%) afectan al pulmón derecho3. La anatomía de la válvula mitral puede contribuir al mecanismo de la EPU, ya que el flujo regurgitante se dirige a las venas pulmonares derechas. Otra posible explicación es el peor drenaje linfático del pulmón derecho por el menor calibre del tronco broncomediastínico en comparación con el mayor tamaño del conducto torácico del lado izquierdo. Además, el crecimiento de las cavidades izquierdas podría dificultar físicamente el flujo de la arteria pulmonar izquierda, reduciendo por tanto e flujo capilar en ese pulmón. Adicionalmente, en este paciente podría haber contribuido un componente posicional, ya que sistemáticamente dormía en decúbito lateral derecho.

La disnea tiene alta sensibilidad en el diagnóstico de IC (100\%), pero muy baja especificidad (17\%). Lo contrario ocurre con el ritmo de galope ventricular como el que presentaba nuestro paciente, que tiene alta especificidad (99\%) pero baja sensibilidad $(24 \%)^{4}$. Tanto los síntomas como los signos clásicos de IC tienen una sensibilidad o especificidad altas, pero no ambas a la vez, y el grado de acuerdo interobservador, en cuanto a la presencia o ausencia de los mismos, es bajo. Por ello, en la práctica se hace necesaria la información objetiva que ofrecen diversas exploraciones complementarias. En este sentido, la posibilidad de realizar en el momento de la valoración inicial del paciente una ecografía pulmonar y un ecocardiograma a pie de cama puede contribuir a seleccionar desde el principio el tratamiento más adecuado para el paciente. En nuestro caso, pudimos objetivar la presencia de líneas B bilaterales en la ecografía pulmonar y una disfunción sistólica severa en ecocardioscopia, cumpliendo por tanto el paciente criterios de IC con FE deprimida según la guía de IC de la ESC ${ }^{2}$

Diversos estudios han demostrado ya la utilidad de la valoración ecográfica a pie de cama en un proceso de descompensación aguda de IC. Lichtenstein et al. ${ }^{5}$ evaluaron el potencial de la ecografía torácica en el diagnóstico de insu- ficiencia respiratoria aguda en pacientes que ingresaban en UCl y demostraron que el hallazgo de líneas B difusas múltiples con deslizamiento pulmonar correspondía con edema pulmonar con sensibilidad del 97\% y especificidad del 95\%.

Como conclusión, podemos establecer que, ante un paciente con un síntoma tan inespecífico como la disnea, el hallazgo de un ritmo de galope ventricular ha demostrado alta especificidad para el diagnóstico de IC con función sistólica deprimida. La ecocardioscopia y la ecografía pulmonar son técnicas diagnósticas que nos permiten conocer la existencia de una cardiopatía estructural y el estado congestivo del paciente, tanto en el momento de la valoración inicial como evolutivamente.

\section{Bibliografía}

1. Registro RECALMIN. Recursos y calidad en Medicina Interna [Internet]. Accesible: https://www.fesemi.org/sites/default/files/documentos/proyectos/recalmin/recalmin-informe-final.pdf

2. Ponikowski P, Voors AA, Anker SD, Bueno H, Cleland JGF, Coats AJS, et al. 2016 ESC Guidelines for the diagnosis and treatment of acute and chronic heart failure: The Task Force for the diagnosis and treatment of acute and chronic heart failure of the European Society of Cardiology (ESC) developed with the special contribution of the Heart Failure Association (HFA) of the ESC. Eur Heart J. 2016 Jul 14; 37(27): 2129-2200. doi: 10.1093/ eurheartj/ehw128.

3. Attias D, Mansencal N, Auvert B, Vieillard-Baron A, Delos A, Lacombe P, et al. Prevalence, characteristics, and outcomes of patients presenting with cardiogenic unilateral pulmonary edema. Circulation. 2010 Sep 14; 122(11): 1109-1115. doi: 10.1161/CIRCULATIONAHA.109.934950.

4. Davie AP, Francis CM, Caruana L, Sutherland GR, McMurray JJV. Assessing diagnosis in heart failure: which features are any use? QJMed. 1997; 90: 335-339.

5. Lichtenstein DA, Mezière GA. Relevance of lung ultrasound in the diagnosis of acute respiratory failure: the BLUE protocol. Chest. 2008 Jul; 134(1): 117-125. doi: 10.1378/chest.07-2800. 